\title{
Cultura organizacional y su relación con los sistemas de gestión: una revisión bibliográfica*
}

[Artículos de revisión]

\author{
Oscar Oswaldo Rincón Rodríguez ${ }^{* *}$ \\ Luperly Aldana Bautista ${ }^{* * *}$
}

Recibido: 7 de julio 2020

Revisado: 8 de marzo de 2021

Aceptado: 15 de marzo de 2021

Citar como:

Rincón Rodríguez, O. O. y Aldana Bautista, L. (2021). Cultura organizacional y su relación con los sistemas de gestión: una revisión bibliográfica. Signos, Investigación en Sistemas de Gestión, 13(2).

https://doi.org/10.15332/24631140.6675

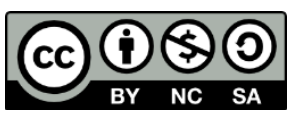

\section{Resumen}

Los sistemas de gestión promueven en la organización el desarrollo de la cultura organizacional con una orientación hacia la mejora continua, por esto, es relevante aplicar procesos de cambio individual y colectivo planeados y dirigidos para lograr el desarrollo de buenas prácticas de

\footnotetext{
*Artículo de revisión.

** Especialista en Derecho Médico Sanitario e ingeniero industrial por la Universidad Incca de Colombia, Bogotá, Colombia. Correo electrónico: oscarrinconr@usantotomas.edu.co, gandhi.o rincon@yahoo.es; ORCID: https://orcid.org/0000-0002-3231-1407; CvLAC: https://scienti.minciencias.gov.co/cvlac/visualizador/generarCurriculoCv.do?cod rh $=0001775867$ ${ }^{* * *}$ Especialista en Dirección para el Desarrollo de la Gestión Pública y administrador de empresas por la Universidad Nacional Abierta y a Distancia, Bogotá, Colombia. Correo electrónico: luperlyaldana@usantotomas.edu.co, luperlyaldana@yahoo.es; ORCID: https://orcid.org/00000001-7063-0217; CVLAC:

https://scienti.minciencias.gov.co/cvlac/visualizador/generarCurriculoCv.do?cod rh=0001769407
}

Signos, Investigación en Sistemas de Gestión

ISSN: 2145-1389 | e-ISSN: 2463-1140 | DOI: https://doi.org/10.15332/24631140

Vol. 13 N.० 2 | julio-diciembre de 2021 
desempeño que hagan eficiente y efectiva la gestión de las funciones.

El estudio expuesto presenta una revisión bibliográfica de las

definiciones e ideas esenciales sobre la transformación, mantenimiento y

diagnóstico de la cultura organizacional. Se realiza una descripción y

yuxtaposición de la información como una estructura comparativa a

partir de las normas técnicas ISO 9000 e ISO 9001, de documentos

académicos y de diversos autores. Esto permiten el análisis,

identificación y clasificación de las dimensiones más significativas

relacionadas con la cultura organizacional y que inciden en su adaptación

e integración. El estudio comparativo concentra y alinea los diferentes

referentes teóricos analizados y relacionados, con los principios de

calidad, y da como resultado la síntesis de las dimensiones que requieren

mayor intervención y que pueden ser incorporados en la cultura

organizacional.

Palabras clave: sistema de gestión, cultura organizacional, gestión del cambio, ISO 9001.

\section{Organizational culture and its relationship with management systems: A literature review}

\section{Abstract}

Management systems promote the organizational culture development within the organization orientated towards continuous improvement. Therefore, it is relevant to apply planned and directed individual and collective change processes to develop good performance practices that make functions management efficient and effective. This study shows a literature review of the definitions and essential ideas about the transformation, maintenance and diagnosis of the organizational culture. A description and juxtaposition of the information is made as a comparative structure based on ISO 9000 and ISO 9001 technical standards, academic documents and various authors. This allows the analysis, identification and classification of the most significant dimensions related to the organizational culture and that affect 
adaptation and integration thereof. The comparative study gathers and aligns the different theoretical references analyzed and related to quality principles, and results in the synthesis of the dimensions requiring greater intervention and that can be incorporated into the organizational culture.

Keywords: management system, organizational culture, change management, ISO 9001.

\section{Cultura Organizacional e sua Relação com Sistemas de Gestão: uma Revisão bibliográfica}

\section{Resumo}

Os sistemas de gestão promovem na organização o desenvolvimento da cultura organizacional voltada à melhoria contínua, portanto, é relevante aplicar processos de mudança individual e coletiva planejados e direcionados para alcançar o desenvolvimento de boas práticas de desempenho que tornem eficiente e eficaz a gestão das funções. O estudo apresenta uma revisão bibliográfica das definições e idéias essenciais sobre a transformação, manutenção e diagnóstico da cultura organizacional. É feita uma descrição e justaposição das informações como estrutura comparativa com base nos padrões técnicos ISO 9000 e ISO 9001, trabalhos acadêmicos e vários autores. Isto permite a análise, identificação e classificação das dimensões mais significativas relacionadas à cultura organizacional e que incidem na sua adaptação e integração. O estudo comparativo concentra e alinha as diferentes referências teóricas analisadas e relacionadas aos princípios de qualidade, e resulta na síntese das dimensões que precisam maior intervenção e que podem ser incorporadas à cultura organizacional.

Palavras-chave: sistema de gestão, cultura organizacional, gestão de mudanças, ISO 9001. 


\section{Introducción}

Las empresas de los sectores productivos y de servicios cuentan con una gran herramienta estratégica y de gestión como son los sistemas de gestión, los cuales les permite potencializar sus fortalezas y oportunidades, y convertirlas en una ventaja competitiva con relación a la demanda de los mercados, de los clientes, de la rentabilidad a alcanzar y de la calidad. Los sistemas de gestión son una herramienta que permite optimizar recursos, reducir costos y mejorar la productividad en una empresa. El sistema de gestión reportará datos en tiempo real que permitirán tomar decisiones para corregir fallos y prevenir la aparición de gastos innecesarios (Fernández, 2016, p. 109). De acuerdo con la Norma técnica ISO 90oo:2015 Sistemas de Gestión de la Calidad - Fundamentos y vocabulario, se define sistema de gestión como un "Conjunto de elementos de una organización interrelacionados o que interactúan para establecer políticas, objetivos y procesos para lograr estos objetivos”.

Los sistemas de gestión están basados en normas técnicas internacionales que permiten controlar distintas facetas en una empresa, como la calidad de su producto o servicio, los impactos ambientales que pueda ocasionar, la seguridad y salud de los trabajadores, la responsabilidad social o la gestión del conocimiento.

Sin embargo, independientemente del nivel de desarrollo estratégico que tenga la organización en cuanto al proceso de incorporación de modelos de gestión, ya se trate de sistemas de gestión de la calidad o de cualquier otro modelo, suelen presentarse obstáculos para su implementación originados en los comportamientos y hábitos arraigados en las personas que conforman la organización, además de la deficiente apropiación de los criterios de cada sistema de gestión, causada por los inadecuados procesos de creación, almacenamiento, transferencia y aplicación del conocimiento. 
Méndez (2006) manifiesta que

[...] los resultados fallidos de la aplicación de tecnologías de gestión pueden explicarse en el contexto de la cultura organizacional, [ya que] se aplican y se adaptan modelos de gestión a personas que comparten una cultura organizacional contraria a los requerimientos para su aplicación. (p. 16)

En tal sentido, es necesario comprender que la cultura de una organización es un factor clave para el éxito o el fracaso en la implementación de los sistemas de gestión; o, como lo afirma Serna (2008), "la cultura corporativa es, por tanto, una de las mayores fortalezas de una organización si coincide con sus estrategias. Pero si esto no ocurre, será una de sus principales debilidades” (p. 133).

La falta de interés de las personas por interiorizar los conceptos de los sistemas de gestión, por generar mejores prácticas de gestión, por impregnarse con voluntad propia de las enseñanzas de los sistemas de gestión y por aplicarlas libremente en el quehacer diario de la sinergia entre proceso y función, acentúan el problema de una implementación fluida y consciente. Esto genera la búsqueda de alternativas para la aplicación de técnicas en el manejo de la "resistencia al cambio", acciones que terminan siendo en muchas ocasiones tan solo un paliativo para las situaciones "problema” presentadas.

Ahora bien, el acercamiento a un concepto general de cultura es muy relevante para entender la sinergia que esta debe generar en los sistemas de gestión, y para esto se cuenta, entre otras, con la investigación de autores como Aguirre (2001), Serna (2008); Nonaka (1995), quienes expusieron: 
[...] la cultura es un constructo cognitivo, un sistema de conocimientos a partir del cual el ser humano, interpreta y regula su forma de comportarse y actuar ante determinadas situaciones organizacionales.

[...] una cultura de calidad empresarial no requiere de supervisores ni de inspectores de calidad. (Rodríguez y Mujica, 2011, p.44)

La misma solo necesita gente que desempeñe día a día sus labores, porque saben que la calidad debe estar presente desde la primera operación. Una cultura de calidad arraigada es una de las mayores fortalezas de una organización, si coincide con las estrategias (Rodríguez y Mujica, 2011).

De acuerdo con González y Fernández (2000), la “cultura organizacional es el conjunto de normas, hábitos y valores que practican individuos de una organización, y que hacen de esta su forma de comportamiento" (p.104).

Varios aportes de diferentes teóricos sobre la cultura organizacional han sido recogidos por el sociólogo Carlos Eduardo Méndez Álvarez (2006), quien destaca los siguientes elementos relevantes para la comprensión de este tema:

La cultura organizacional es una construcción colectiva determinada por la acción social. De esta forma la identidad y pertenencia a la organización llevan al hombre de la organización a creer y tener un comportamiento similar al de otros miembros y que han consolidado en el tiempo. Por ello, tiene un componente emocional en el cual el individuo conoce formas de conducta que orientan su comportamiento y le crean expectativas frente al de los otros miembros de la organización, reduciendo la incertidumbre y reafirmando la seguridad en sus relaciones enmarcadas en el sistema social y reflejadas en el sistema de personalidad. (p. 88) 
La cultura organizacional se define como una percepción compartida por los miembros de un grupo sobre los valores, creencias, principios y normas que influyen en los aspectos de la vida laboral y rigen la toma de decisiones (Cameron y Quinn, 2006; Schein 1988; Soemaryani y Rakhmadini, 2013).

Este estudio comparativo concentra y alinea los diferentes referentes teóricos relacionados con la cultura organizacional y, a su vez, con los principios de la calidad de la norma técnica internacional ISO 9001:2015. Con esto pretende analizar, identificar y clasificar las dimensiones más significativas, y dar como resultado la síntesis de las dimensiones que requieren mayor intervención y deben ser incorporados en la cultura organizacional para que incidan en su adaptación e integración.

Con el presente estudio comparativo se pretende identificar la complementariedad entre la cultura organizacional en sistemas de gestión y los principios de la calidad de la norma técnica internacional ISO 9001:2015: enfoque al cliente, liderazgo, compromiso de las personas, enfoque a procesos, mejora, toma de decisiones basada en la evidencia, gestión de las relaciones y algunas dimensiones influyentes en el actuar de las personas, a través de las cuales se concretará una activa y voluntaria participación en la calidad organizacional.

\section{Metodología}

La metodología aplicada en el presente estudio fue la revisión sistemática exploratoria: "síntesis de la evidencia sobre un tema relacionado sobre un tema específico que describe el conocimiento existente sobre el mismo. Sirven para generar hipótesis, establecer líneas de investigación, o como base para la elaboración de informes técnicos" (Manchado, 2009). Se realizó una revisión sistemática exploratoria sobre la cultura organizacional y los sistemas de gestión, que arrojó consideraciones 
específicas entre dimensiones inherentes a la cultura organizacional y los sistemas de gestión.

Los documentos consultados para la revisión bibliográfica se extrajeron de bases de datos documentales, de revistas académicas, artículos científicos, diferente material archivado y trabajos académicos de varias universidades de América Latina y el Caribe, y otros brindados por la Universidad Santo Tomás, CRAI-USTA, con la revista Signos y las herramientas de investigación como Scopus, ScienceDirect y Redalyc. Los descriptores utilizados fueron "sistema de gestión”, "cultura organizacional”, "gestión del cambio", "ISO 9001". En una primera fase, los documentos detectados se limitaron temporalmente entre los años 2004 a 2019, se identificaron 150, de los cuales se seleccionaron 33 con criterios de calidad e inclusión alta, esto en razón que consideraban referentes teóricos importantes relacionados con la revisión bibliográfica en cuestión, desarrollaron análisis pertinentes en cuanto a los descriptores utilizados y aportaban componentes concluyentes sobre la formación de la cultura organizacional. En la segunda fase, se consolidaron los documentos seleccionados en una matriz de revisión de literatura, consignando los resúmenes, marcos teóricos, metodologías, resultados concluyentes y la mención sobre la cultura organizacional, sistema de gestión y los siete principios de gestión de la calidad de la norma técnica internacional ISO 9001, para hacerlos parte del análisis y cumplir con el objetivo de la presente investigación bibliográfica.

Finalmente, se desarrolló una última fase de análisis con la bibliografía detectada, al identificar la coincidencia de las temáticas estructurales relevantes de los documentos contra la cultura organizacional en sistemas de gestión al extraer los aspectos con mayor intervención o convergencia entre los documentos analizados, en una matriz de convergencia, se 
encontró que las más representativas en su orden fueron: comunicación, aprendizaje, gestión del cambio y valores organizacionales.

\section{Resultados y discusión}

En la revisión bibliográfica realizada, se pudieron identificar temáticas de convergencia, extraídas de los artículos consultados, luego de lo cual, bajo un consenso de coherencia relacional, se identificaron las que más aportaban a la formación de una cultura organizacional en sistemas de gestión, como también la coincidencia conceptual con los principios de calidad de la norma técnica internacional ISO 9001:2015.

La cultura organizacional es entendida como

[...] el patrón de supuestos básicos que un determinado grupo ha inventado, descubierto o desarrollado al aprender a hacer frente a los problemas de adaptación externa e integración, y que ha funcionado suficientemente bien como para considerarlo válido, y por tanto para enseñarlo a los nuevos miembros como la forma correcta de percibir, pensar y sentir en relación con esos problemas. (Schein, 1992, p. 12)

La cultura organizacional es una temática que ha tenido gran relevancia e importancia, aun desde los años setenta y ochenta. Es necesario que la cultura organizacional esté presente en cada organización, ya que esta es la encargada de fomentar un mayor desempeño tanto individual como colectivo de los trabajadores (Robbins, 2005, citado por Miquilena y Paz, 2008).

La cultura es un determinante del desempeño, ya que impacta no solo los resultados directos y la eficiencia de una organización, sino también la salud, el entusiasmo, el compromiso y la flexibilidad de su personal. El desempeño puede medirse de acuerdo con medidas financieras y con no financieras (Toca, 2009, p. 119-125). 
Schein (1988) introdujo los conceptos de presunciones y creencias para explicar de forma más amplia el significado que para la organización tienen el concepto de cultura; la define entonces como "respuestas que ha aprendido el grupo ante sus problemas de subsistencia en su medio externo y ante sus problemas de integración interna" (Schein, 1988, p. 24). Este nivel de presunciones y creencias que comparten los grupos en la organización corresponde a la esencia misma de la cultura. La cultura debe ser vista como las dinámicas de cada organización para establecer la identidad, las relaciones, las actitudes y los valores como aspectos que impactan el direccionamiento de la organización, en sinergia con el entorno que los individuos han experimentado e implementado para adaptarse a los cambios organizacionales.

Como sugiere Schein (1988): "es un concepto formal para la cultura organizacional, en tal sentido es un producto aprendido de la experiencia, y por tanto algo localizable solo allí, donde exista un grupo definible y poseedor de una historia significativa” (pp. 24-25).

Las personas, eje principal del cambio y generadoras de la cultura de la calidad en una organización, poseen un elemento psicológico constitutivo de la conducta que inciden en el proceso de implementación de las normas técnicas internacionales ISO. Este campo psicológico actúa en las organizaciones y, por lo tanto, deben descifrarse las variables que inciden en la diversidad de personas integrantes de las organizaciones, a las cuales hay que alinear con el fin de generar sinergias para "obtener una conformidad con un desempeño esperado; ya sea en términos de la gestión de calidad o bien en términos del desempeño competente" (Andino, 1999; Peach, 1999).

La cultura organizacional se convierte en una pieza estratégica dentro de las organizaciones, genera que sus miembros desarrollen un sentido de 
identidad con la empresa, a través de formas de pensar, creencias y valores, incrementando así sus ventajas competitivas e impactando en el desempeño de esta (Etkin, 2000).

Mediante una cultura organizacional cimentada en el respeto por la persona, que contribuya al crecimiento personal, profesional, familiar y social, en interacción con el entorno, se conseguirá que esta impacte sobre el rendimiento o desempeño de los que conforman una organización. Que incidan en el sentido de pertenencia, en el logro de objetivos y resultados con eficiencia y, por defecto, generará un ambiente organizacional sano y un alto nivel de bienestar del personal, con intervenciones positivas en las emociones, en la salud, en el entusiasmo, el compromiso y la flexibilidad de su personal.

Sin dejar olvidado este precepto, se busca fortalecer el liderazgo en el talento humano en todos los niveles de la organización, optimizando y fortaleciendo las competencias personales y laborales de las personas; esto con el propósito de alcanzar estándares de calidad, eficiencia y efectividad en los sistemas de gestión, para alcanzar los resultados y niveles de desempeño trazados por la organización. Esto da relevancia al poder que las personas ejercen sobre la implementación de los sistemas de gestión, mediante su empoderamiento, interpretación, auto regulación, comportamiento y actuación ante la toma de conciencia para incidir en la metodología de implementación y en la generación de una cultura organizacional que incorpore el respeto por el recurso humano.

La formación del personal es importante, ya que mejora el talento humano, eje dinamizador de la cultura organizacional en los sistemas de gestión, aumenta la calidad de sus habilidades laborales ante los desafíos, para lograr la satisfacción de los clientes y de las partes interesadas. El empoderamiento de las personas para alcanzar un estilo de vida basado 
en los sistemas de Gestión debe ser considerado como una necesidad individual, social y organizacional.

Para impulsar y concretar un cambio en la cultura de cualquier organización es indispensable seguir algunas reglas y recomendaciones establecidas por diversos autores, de las cuales se rescatan las que definió Jurán (1990):

1. Involucrar a la alta dirección de la organización. Sin su apoyo poco o nada podrá concretarse.

2. Impulsar un continuo aprendizaje y capacitación. Dirigido a todos los niveles tanto operativos como del equipo de la alta dirección.

3. Recompensas, reconocimiento y celebración.

4. Procesos organizativos que permitan la construcción de equipos para la mejora de la calidad.

5. Reclutar, seleccionar y socializar empleados orientados a la calidad.

6. Adicionalmente el mismo autor proporciona unas reglas necesarias para lograr transformar la cultura de alguna organización.

7. Proveer la participación de la organización durante la planeación y ejecución del cambio.

8. Proveer suficiente tiempo para que la organización evalúe las ventajas del cambio cultural en la organización.

9. Comenzar gradualmente, de forma fluida y realizar pruebas piloto.

10. Crear un clima social favorable, para lo cual es indispensable la participación del equipo directivo de alto nivel.

11. Responder positivamente a los problemas suscitados por los empleados.

12. Trabajar con el líder reconocido de la cultura que a menudo es informal.

13. Tratar a las personas con dignidad y hacer los cambios constructivos. 
La revisión bibliográfica generada sobre la cultura organizacional y los sistemas de gestión arrojó como resultado la síntesis de doce dimensiones que han requerido mayor intervención y que han debido o han sido incorporados en la cultura organizacional de diferentes tipos de empresas, y que resultaron ser las más nombradas o estudiadas en los 33 artículos revisados. De mayor a menor participación resultó lo siguiente: comunicación en 30 artículos (91\%), aprendizaje en 29 artículos (88 \%), gestión del cambio en 27 artículos (82\%), cultura organizacional en 26 artículos (79\%), valores organizacionales en 25 artículos (76\%), tecnología en 24 artículos (73\%), participación de todos los niveles en 23 artículos (70 \%), reconocimiento en 22 artículos (67\%), gestión del conocimiento en 21 artículos (64\%), cultura de calidad en 20 artículos (61\%), clima organizacional en 15 artículos (45\%) y toma de conciencia en 14 artículos (42\%) (Ver figura 1). 
Figura 1. Dimensiones incidentes en la cultura organizacional de sistemas de gestión

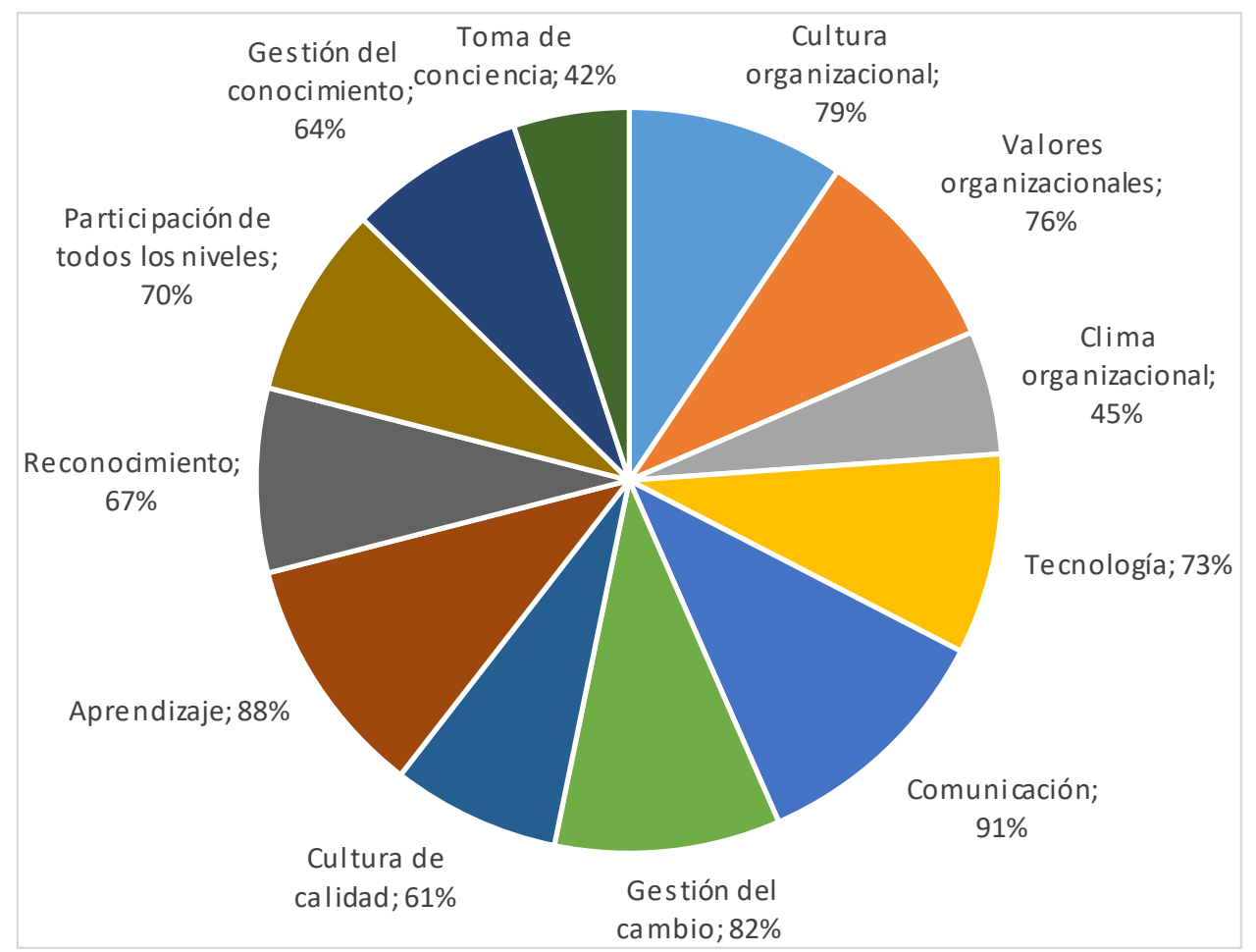

Fuente: elaboración propia (2020).

Como un marco conceptual, se presentan los postulados propuestos por los autores consultados respecto de las dimensiones identificadas como de mayor incidencia:

Cultura organizacional: dinámicas de cada organización para establecer la identidad, las relaciones, las actitudes y los valores como aspectos que impactan el direccionamiento de la empresa.

Valores organizacionales: orientan las acciones a seguir por parte de cada uno de los participantes, lo que asegura que la suma de sus conductas, actuaciones, sean guiadas por el compromiso, la armonía, la reputación y la trayectoria de la organización. 
Clima organizacional: cualidad relativamente duradera del ambiente interno de una organización que experimentan sus miembros, influye en su comportamiento y puede describirse en términos de los valores de una serie particular de características (o atributos) de la organización.

Tecnología: se define como el conjunto de conocimientos y técnicas que, aplicados de forma lógica y ordenada, permiten al ser humano modificar su entorno material o virtual para satisfacer sus necesidades, es decir, un proceso combinado de pensamiento y acción con la finalidad de crear soluciones útiles.

Comunicación: la comunicación hace posible difundir y transmitir la información de calidad que se genera en toda la entidad, tanto entre dependencias como frente a los grupos de valor. Contar con servidores públicos bien informados sobre cómo opera la entidad y con ciudadanos bien informados sobre cómo hacer efectivos sus derechos, fomenta la eficiencia, la eficacia, la calidad y la transparencia en la gestión pública, la rendición de cuentas por parte de sus directivos.

Gestión del cambio: la gestión del cambio abarca estrategias de administración, métodos y tecnología para nivelar el capital intelectual y el know-how, con el fin de lograr ganancias en rendimiento y competitividad humana.

Cultura de calidad: es un ambiente en el que los empleados no solo siguen las pautas de calidad, sino que también ven a los demás de manera sistemática, toman medidas enfocadas en la calidad, escuchan a otros hablar sobre calidad y sienten la calidad a su alrededor.

Aprendizaje: es la adquisición de conocimiento sobre los sistemas de gestión, a través de mecanismos funcionales y diversos que permiten que las personas apliquen en su diario vivir organizacional los conceptos y la estructura metodológica del sistema, en las prácticas laborales.

Signos, Investigación en Sistemas de Gestión

ISSN: 2145-1389 | e-ISSN: 2463-1140 | DOI: https://doi.org/10.15332/24631140

Vol. 13 N. ${ }^{\circ} 2$ | julio-diciembre de 2021 
Reconocimiento: representa el grado de valoración, distinción o agradecimiento a las personas que contribuyen en forma vivencial y voluntaria a la implementación y mantenimiento de los sistemas de gestión y a los objetivos de la organización. La organización debe desarrollar estrategias motivacionales con el fin de que las personas sientan el reconocimiento.

Participación de todos los niveles. Es el involucramiento de todas las personas pertenecientes a la organización, en la implementación y mantenimiento de los sistemas de gestión, mediante acciones sinérgicas y con valor agregado.

Gestión del conocimiento: es el proceso organizacional dirigido a adquirir, transferir, mantener, aplicar y conservar el conocimiento entre las personas que conforman una organización, potencializando el desempeño de las personas y de la organización.

Toma de conciencia: es un estado cognitivo que permite que una persona interprete y actúe responsablemente, de acuerdo con unos estímulos externos que forman cierta realidad, en este caso los sistemas de gestión. Se refleja en el actuar en mayor o menor grado de aplicabilidad. Y respecto a los principios de la norma técnica internacional ISO 9001:2015, en la muestra de los 33 artículos analizados se presenta en orden de porcentaje, su citación en ellos: liderazgo en 27 artículos (82 \%), compromiso de las personas en 27 artículos (82\%), mejora continua en 27 artículos (82 \%), toma de decisiones en 25 artículos (76\%), enfoque al cliente en 21 artículos (64\%), enfoque a procesos en 21 artículos (64\%) y gestión de las relaciones en 21 artículos (64 \%) (ver figura 2). 
Figura 2. Principios de calidad norma técnica internacional ISO 9001:2015

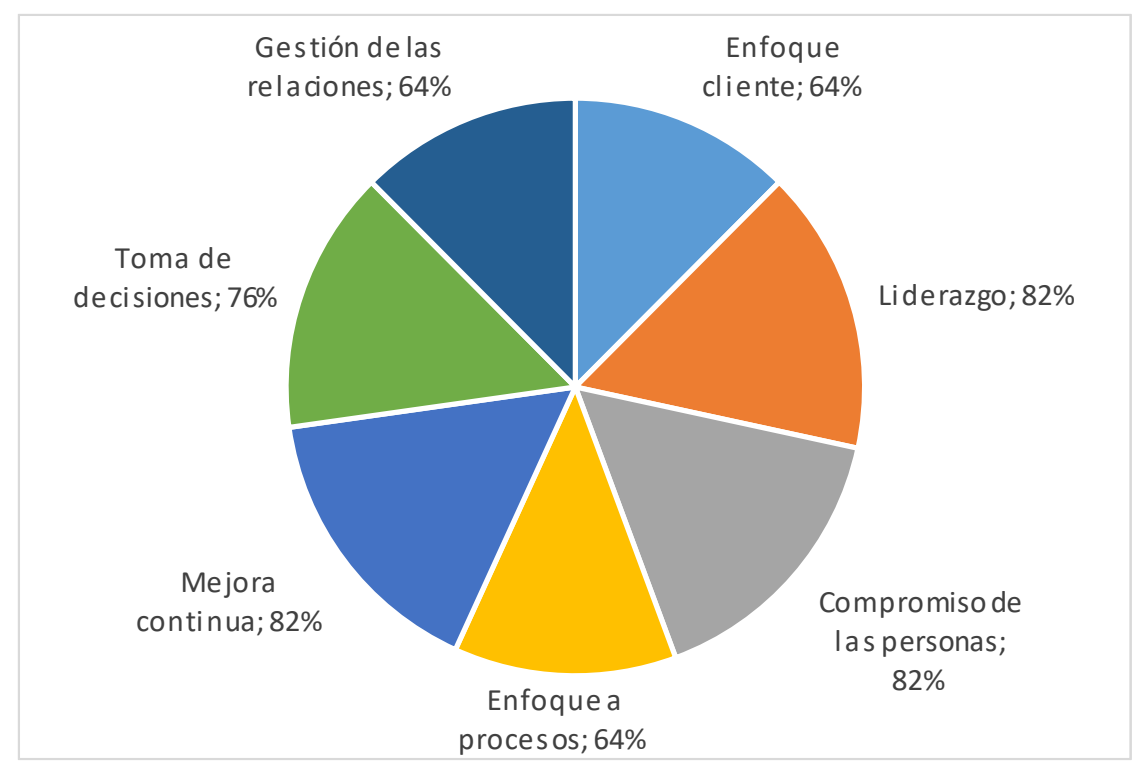

Fuente: elaboración propia (2020).

La conceptualización de los principios ha sido:

Enfoque al cliente: las organizaciones dependen de sus clientes y, por lo tanto, deben comprender las necesidades actuales y futuras de los clientes, satisfacer los requisitos de los clientes y esforzarse en exceder sus expectativas.

Liderazgo: los líderes establecen la unidad de propósito y la orientación de la organización. Ellos deberían crear y mantener un ambiente interno en el cual el personal pueda llegar a involucrarse totalmente en el logro de los objetivos de la organización.

Participación del personal: el personal, a todos los niveles, es la esencia de una organización y su total compromiso posibilita que sus habilidades sean usadas para el beneficio de la organización.

Enfoque basado en procesos: un resultado deseado se alcanza más eficientemente cuando las actividades y los recursos relacionados se gestionan como un proceso. 
Mejora: la mejora continua del desempeño global de una organización debería ser un objetivo permanente de esta. Esa mejora continua de los procesos se consigue siguiendo el ciclo PHVA de Deming (1989): planificar, hacer verificar, actuar, para mejorar.

Enfoque basado en hechos para la toma de decisión. Las decisiones se basan en el análisis de los datos y la información.

Gestión de las relaciones: la correcta gestión de las relaciones que la organización tiene para con la sociedad, los socios estratégicos y los proveedores contribuyen al éxito sostenido de la organización.

Algunos de los trabajos considerados analizan y describen elementos y características de los sistemas de gestión de la calidad y sus principios sin enfocarse en normativas o modelos particulares; otros, en el tema de las dimensiones, exponen las posibles incidencias en la cultura organizacional, es decir, lo que más ha provocado deficiencias en la consolidación de una cultura organizacional en sistemas de gestión.

\section{Conclusiones}

Después del análisis de las referencias bibliográficas categorizadas, se concluye que las dimensiones y principios de la gestión de la calidad más analizados, más citados y con mayor intervención sobre los artículos consultados, en los que se expusieron las ideas y aspectos relevantes, comprendieron las dimensiones de comunicación (91\%), aprendizaje (88 \%), gestión del cambio (82\%), e igualmente para los principios de calidad fueron el liderazgo ( $82 \%$ ), compromiso de las personas ( $82 \%$ ) y mejora continua ( $82 \%$ ).

También se identificaron unas temáticas de convergencia (tendencia a reunirse en un punto), resultado de lo tratado en los artículos consultados y que, en consenso, aportaban a la formación de una cultura 
organizacional en sistemas de gestión y, igualmente, a la coincidencia conceptual con los principios de calidad de la norma técnica internacional ISO 9001:2015. Se encontró que la comunicación, el aprendizaje, la cultura y la gestión del cambio poseen elementos de coherencia con los planteamientos de los principios de liderazgo, participación del personal y mejora. De este análisis se distingue un eje dinamizador integrado entre principios y dimensiones que lo constituye, las personas de una organización, las que ejercen gran relevancia sobre la implementación de los sistemas de gestión, mediante su empoderamiento, interpretación, autoregulación, comportamiento y actuación ante la toma de conciencia para incidir en la metodología de implementación y en la generación de una cultura organizacional.

En síntesis, la convergencia de dimensiones apuntó al componente humano que, a través de la comunicación, el aprendizaje y la gestión del cambio, incide en la formación de una Cultura organizacional en los sistemas de gestión.

La cultura organizacional en sistemas de gestión mantiene una relación estrecha con los principios de calidad del liderazgo, compromiso de las personas y mejora continua, y estos a su vez confluyen al componente humano como factor clave en la estructura de alto nivel de la norma técnica ISO 9001, liderazgo y compromiso [5.1], roles, autoridad y responsabilidad [5.3], conocimientos de la organización [7.1.6], competencias [7.2], comunicación [7.4], toma de conciencia [7.3] y por ende en la formación de una cultura organizacional sólida.

Encontramos que entre los diferentes autores existen aspectos claves que convergen sobre los preceptos de Jurán (1990).

Para reforzar el cambio o formación de la cultura organizacional en sistemas de gestión es indispensable implementar las reglas y 
recomendaciones establecidas por Jurán (1990), y que coinciden con algunas de las dimensiones identificadas en esta revisión bibliográfica, como son "involucrar a la alta dirección, impulsar un continuo aprendizaje, implementar recompensas, reconocimiento y celebración, proveer la participación de la organización, crear un clima social favorable y tratar a las personas con dignidad" (p. 306). Se acentúa la estrecha relación con el componente humano en las organizaciones.

Serna (2008) plantea que "la cultura corporativa es, por tanto, una de las mayores fortalezas de una organización si coincide con sus estrategias. Pero si esto no ocurre, será una de sus principales debilidades” (p. 133). Este argumento respalda lo encontrado en el análisis referido en cuanto a que, si la organización implementa estrategias dirigidas a la comunicación, el aprendizaje, la cultura y la gestión del cambio, dimensiones a ser apropiadas por el talento humano, se contribuirá a fortalecer la cultura organizacional, en este caso en los sistemas de gestión. Con las estrategias, la organización podrá gestionar los riesgos que inciden en su implementación, originados en comportamientos y hábitos arraigados en las personas, además de la deficiente apropiación de los criterios propios de cada sistema de gestión, causada por los inadecuados procesos de creación, almacenamiento, transferencia, interpretación y aplicación del conocimiento.

Los posibles riesgos por materializarse, producto del intelecto humano, están relacionados con lo expuesto por Rodríguez y Mujica (2011) en el estudio Influencia de la metodología de implantación de los sistemas de calidad en la cultura de la PYMES el cual se soportó en varios autores, como Aguirre (2004), Serna (2008) y Nonaka (1995):

[...] la cultura es un constructo cognitivo, un sistema de conocimientos a partir del cual el ser humano, interpreta y regula su forma de 
comportarse y actuar ante determinadas situaciones organizacionales. (Rodríguez y Mujica, 2011, p. 44)

En cuanto a la dimensión de gestión del cambio, algunos documentos analizados indujeron a la teoría de que los integrantes de una organización cultivan o marchitan el proceso de formación de la identidad, las relaciones, las actitudes y los valores organizacionales, causado en cierta medida por un proceso fallido de adaptación del cambio. Esto constituyen otro riesgo latente que impacta el direccionamiento de la organización. La falta de interés de las personas por impregnarse con la voluntad propia de las enseñanzas de los sistemas de gestión y por aplicarlas libremente en el logro de los objetivos trazados acentúan el problema de una implementación fluida y consciente, lo que genera la búsqueda de alternativas para la aplicación de técnicas en el manejo de la resistencia al cambio.

$\mathrm{Al}$ respecto, Méndez (2006) manifiesta que

[...] los resultados fallidos de la aplicación de tecnologías de gestión pueden explicarse en el contexto de la cultura organizacional, [ya que] se aplican y se adaptan modelos de gestión a personas que comparten una cultura organizacional contraria a los requerimientos para su aplicación. (p. 16)

Ahora bien, la transformación voluntaria y armoniosa de los individuos de una organización frente a los cambios en el ser, pensar y hacer del devenir laboral debe realizarse según los preceptos expuestos por los autores García et ál. (2011), quienes consideran que "La capacidad para adaptarse al cambio es considerada como uno de los retos que enfrentan las organizaciones cuando ven la necesidad de modificar sus esquemas de trabajo y optimizar la gestión de la empresa” (p. 129). 
Todo proceso de gestión del cambio en la cultura organizacional deberá valerse de algunas reglas y recomendaciones como las expuestas por Jurán (1990), y que están relacionadas con las dimensiones resultantes de esta revisión bibliográfica: comunicación, aprendizaje y gestión del cambio. Ahora bien, Casas y Roca (2016) relacionan que una clara oportunidad de mejorar los niveles de participación de las personas en una organización, en el desarrollo de los sistema de gestión, (desde la comprensión, hasta la toma de conciencia, compromiso, sentido de pertenencia) es a través de acciones orientadas a materializar la comunicación desde una perspectiva estratégica, integradora y holística, cultural, operativa y de crecimiento individual y colectivo, para lograr fortalecer aspectos como la cultura del autocontrol, el liderazgo y las capacidades de aprendizaje. Los sociólogos Peter Berger y Thomas Luckmann (1999) plantean desde el constructivismo social que la comunicación es realmente significativa para construir la realidad social. Por un lado, se debe tener en cuenta que la comunicación, inmersa en las relaciones personales, es la base de los consensos y, al mismo tiempo, a través de la comunicación se fijan las pautas de la convivencia y participación. En consonancia con lo anterior, la norma técnica internacional ISO 9001:2015, en su numeral 7.4, cita que la organización debe determinar las comunicaciones internas y externas con el fin de asegurar que los integrantes de la organización sean conscientes de la pertinencia de las comunicaciones y de cómo contribuyen al logro de los objetivos propuestos por la entidad.

Respecto a la dimensión aprendizaje, Casallas y Gaona (2013), en su trabajo de grado resaltan que:

En la adopción de un modelo de gestión se debe contemplar unas condiciones que inciden en los comportamientos y actitudes de las personas de una institución como miembros que integran una cultura 
organizacional, se imparte a través del aprendizaje a través de la socialización al individuo que forma parte de la organización, debe ser impartida de manera dinámica y puede ser transformada. (p. 17)

Por lo cual es prudente considerar y enfatizar el recurso humano como un eje fundamental en el logro de las estrategias y objetivos que se propone la organización; por esto son vitales los procesos de capacitación y desarrollo de personal, teniendo en cuenta que dichas prácticas tienen un impacto significativo en el logro de los objetivos y estrategias planteadas.

Finalmente, para lograr la integración entre principios de la calidad y dimensiones de la cultura organizacional, las organizaciones deben asegurarse de que la forma de operar sea justa y equitativa. Cuando hablamos de equidad nos referimos a la necesidad de incluir en el proceso a todas las personada afectadas, a fin de que conozcan de primera mano los objetivos y estrategias planteadas. Pero esta estrategia requiere una gestión de cambio, por lo cual nuestra investigación sugiere que puede lograrse mediante el liderazgo para inclinar la balanza. Al identificar conscientemente las barreras para la ejecución de la estrategia y concentrarse en los factores de influencia, es posible derrumbar esas barreras y hacer realidad el cambio estratégico, la preparación y la consolidación de la cultura de calidad como estilo de vida organizacional. Este es el eje de éxito que asegura la participación de las personas, que son el corazón de las entidades o empresas y quienes independientemente al nivel organizacional que pertenezcan, mueven las organizaciones.

\section{Referencias}

Aguirre, J. M. (2001). Nuevas fronteras y escenarios culturales en la Sociedad de la Información. En J. Aguirre y M. Álvarez (eds.), Periodismo y fronteras culturales. Tauro. 
Andino, M. (1999). La implementación de las normas ISO 9000 y sus efectos positivos en el departamento de control de calidad. Experiencia de Famosa en El Salvador, Centroamérica. Ciencia y tecnología, 2(3).

Barahona P., J. A., y Rodríguez A., D. (2015). La cultura organizacional, un camino para humanizar la implementación del sistema de gestión de calidad - ISO 9001:2008. Signos, Investigación en Sistemas de Gestión,00207(2), 19-29. https://doi.org/10.15332/s214.5-1389.2015.0002.01

Berger, P. y Luckmann, T. (1999). La construcción social de la realidad. Amorrortu Editores.

Cameron, K. S. y Quinn, R. E. (2006). Diagnosing and Changing Organizational Culture: Based on the Competing Values Framework. Jossey-Bass.

Casas H., A. D. P., y Roca M., J. J. (2016). Diagnóstico de la comunicación interna y la participación en sistemas de gestión de la calidad en dos entidades del sector público colombiano. Signos, Investigación en Sistemas de Gestión, 8(2), 39-63. https://doi.org/10.15332/s2145-1389.2016.0002.02

Casallas, R. P., y Gaona, P. J. M. (2013). La cultura organizacional, factor crítico de éxito en procesos de gestión de la calidad y de acreditación institucional. Signos, Investigación en Sistemas de Gestión, 5(1), 15-30. https://doi.org/10.15332/s21451389.2013 .0001 .01

Deming, W. E. y Medina, J. N. (1989). Calidad, productividad y competitividad: la salida de la crisis. Díaz de Santos.

Etkin, J. y Schvarstein, L. (2000). Identidad de las organizaciones, invariancia y cambio. Paidós.

Etkin, J. (2000). Gestión de la complejidad en las organizaciones. La estrategia frente a lo imprevisto y lo impensado. Ediciones Granica S. A.

Fernández, L. G. (2016). Normativa y política interna de gestión ambiental de la organización. Editorial Tutor Formación.

García, M., Rojas, M. F. y Díaz, S. (2011). Relación entre el cambio organizacional y la actitud al cambio en trabajadores de una empresa de Bogotá. Diversitas:

Perspectivas en Psicología, 7(1), 129.

https://www.redalyc.org/articulo.oa?id=67922583009

Signos, Investigación en Sistemas de Gestión

ISSN: 2145-1389 | e-ISSN: 2463-1140 | DOI: https://doi.org/10.15332/24631140

Vol. 13 N.o 2 | julio-diciembre de 2021 
González, B. J. A. (2016). Metodología para fortalecer la participación del personal en el Sistema de Gestión de Seguridad y Salud en el Trabajo en una empresa del sector aeronáutico. Signos, Investigación en Sistemas de Gestión, 8(1), 67-85. https://revistas.usantotomas.edu.co/index.php/signos/article/view/3789/3734

Gonzáles, A., y Fernández, E. M. (2000). La cultura de la organización en la gestión total de la calidad. Ensaios e Ciência: Ciências Biológicas, 4(3), 104. https://www.redalyc.org/articulo.oa?id=26040307\&idp=1\&cid=1952012

Jurán, M. (1990). Jurán y el liderazgo para la Calidad. Díaz de Santos.

Manchado Garabito, R., Tamames Gómez, S., López González, M., Mohedano Macías, L., D’Agostino, M., y Veiga de Cabo, J. (2009). Revisiones Sistemáticas Exploratorias. Medicina y Seguridad del Trabajo, 55(216), 14. http://scielo.isciii.es/scielo.php?script=sci arttext\&pid=S0465546X2009000300002

Méndez Álvarez, C. E. (2006). Transformación cultural en las organizaciones. Un modelo para la gestión del cambio. Editorial Universidad del Rosario.

Miquilena, D., y Paz, A. (2008). La cultura como fuente de compromiso del capital humano en las organizaciones modernas. http://www.publicaciones.urbe.edu/index. $\mathrm{php} /$ forumhumanes/article/viewArticle/475/1170

Nonaka, I. y Takeuchi, H. (1995). The Knowledge-Creating Company- How Japanese Companies Create the Dynamics of Innovation. Oxford University.

Norma Técnica ISO 9000. (2015). Sistemas de Gestión de la Calidad - Fundamentos y vocabulario.

Peach, R. (1999). Manual de ISO 9ooo. Graw Hill.

Pedraza-Álvarez, L., Obispo-Salazar, K., Vásquez-González, L., y Gómez-Gómez, L. (2015). Cultura organizacional desde la teoría de Edgar Schein: Estudio fenomenológico. Revista Clío América, 9(17), 17-25. http://revistas.unimagdalena.edu.co/index.php/clioamerica/article/view/1462

Robbins, S. (1999). Comportamiento organizacional. Prentice Hall.

Robles, A. C. (2018). Diseño y validación de un instrumento de cultura organizacional para empresas medianas. Nova Scientia.

Signos, Investigación en Sistemas de Gestión

ISSN: 2145-1389 | e-ISSN: 2463-1140 | DOI: https://doi.org/10.15332/24631140

Vol. 13 N.0 2 | julio-diciembre de 2021 
Rodríguez, A. y Mujica, M. (2011). Influencia de la metodología de implantación de los sistemas de calidad en la cultura de la PyMEs [tesis de maestría]. Universidad de Carabobo.

Rodriguez, A. D., y Barahona, P. Y. A. (2015). Propuesta metodológica para el mejoramiento de la cultura organizacional en la implementación del SGC ISO 9001:2008 [proyecto de pasantía]. Universidad Francisco José de Caldas.

Rodríguez, R. L. A. y Lombana M., A. C. (2015). Herramienta coaching como factor motivacional en la implementación de la NTC ISO 9001:2008 en el área de biblioteca de la Fundación Universitaria de Ciencias de la Salud (FUCS). Signos, Investigación en Sistemas de Gestión, 7(2), 61-71. https://doi.org/10.15332/s21451389.2015.0002.04

Schein, E. H. (1992). Organizational Culture and Leadership. CA Jossey Bass.

Schein, E. H. (1988). La cultura empresarial y el liderazgo. Una visión dinámica. Plaza \& Janes Editores. P.

Serna, H. (2008). Gerencia, Estratégica, Planificación y Gestión: Teoría y Metodología. 3 R Editores.

Soemaryani, I. y Rakhmadini, D. (2013). Work Life Balance and Organizational Culture in Creating Engagement and Performance. International Journal of Innovations in Business, 2(4),327-349.

Toca, C. E. (2009). Asuntos teóricos y metodológicos de la cultura organizacional. Civilizar, 9(17), 117-136. 